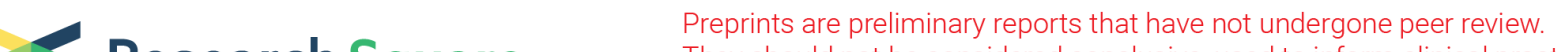 Research Square They should not be considered conclusive, used to inform clinical practice, or referenced by the media as validated information.
}

\section{A Fast Extraction-Free Isothermal LAMP Assay for Detection of SARS-CoV-2 in Resource-Limited Settings}

\section{Kathleen Gärtner ( $\sim$ k.gartner@ucl.ac.uk )}

University College London Institute of Child Health https://orcid.org/0000-0003-2221-6251

\section{Harry Meleke}

University of Malawi Kamuzu College of Nursing: University of Malawi College of Medicine

\section{Mercy Kamdolozi}

University of Malawi Kamuzu College of Nursing: University of Malawi College of Medicine

\section{David Chaima}

University of Malawi Kamuzu College of Nursing: University of Malawi College of Medicine

\section{Lyson Samikwa}

University of Malawi Kamuzu College of Nursing: University of Malawi College of Medicine

\section{Mary Paynter}

St George's Hospital Medical School: St George's University of London

\section{Maggie Nyirenda Nyang'Wa}

University College London Institute of Child Health

\section{Elaine Cloutman-Green}

Great Ormond Street Hospital NHS Trust: Great Ormond Street Hospital For Children NHS Foundation Trust

\section{Eleni Nastouli}

UCLH: University College London Hospitals NHS Foundation Trust

\section{Nigel Klein}

University College London Institute of Child Health

\section{Tonney Nyirenda}

University of Malawi Kamuzu College of Nursing: University of Malawi College of Medicine

\section{Chisomo Msefula}

University of Malawi Kamuzu College of Nursing: University of Malawi College of Medicine

\section{Dagmar G. Alber}

University College London Institute of Child Health

\section{Research Article}

Keywords: SARS-CoV-2, qRT-PCR, extraction-free LAMP, resource-limited settings 
Posted Date: January 27th, 2022

DOI: https://doi.org/10.21203/rs.3.rs-1276926/v1

License: (c) (i) This work is licensed under a Creative Commons Attribution 4.0 International License. Read Full License 


\section{Abstract \\ Background}

To retain the spread of SARS-CoV-2, fast, sensitive and cost-effective testing is essential, particularly in resource limited settings (RLS). Current standard nucleic acid-based RT-PCR assays, although highly sensitive and specific, require transportation of samples to specialised laboratories, trained staff and expensive reagents. The latter are often not readily available in low- and middle-income countries and this may significantly impact on the successful disease management in these settings. Various studies have suggested a SARS-CoV-2 loop mediated isothermal amplification (LAMP) assay as an alternative method to RT-PCR.

\section{Methods}

Four previously published primer pairs were used for detection of SARS-CoV-2 in the LAMP assay. To determine optimal conditions, different temperatures, sample input and incubation times were tested. Ninety-two extracted RNA samples from St. George's Hospital, London, 10 non-extracted nasopharyngeal swab samples from Great Ormond Street Hospital for Children, London, and 92 non-extracted samples from Queen Elisabeth Central Hospital (QECH), Malawi, which have previously been tested for SARS-Cov2 by $\mathrm{qRT}-\mathrm{PCR}$, were analysed in the LAMP assay.

\section{Results}

In this study we report the optimisation of an extraction-free colourimetric SARS-CoV-2 LAMP assay and demonstrated that a lower limit of detection between 10-100 copies/ $\mu \mathrm{L}$ of SARS-CoV-2 could be readily detected by a colour change of the reaction within as little as $30 \mathrm{~min}$. We further show that this assay could be quickly established in Malawi, as no expensive equipment is necessary. We tested 92 clinical samples from QECH and showed the sensitivity and specificity of the assay to be $98.4 \%$ and $86.7 \%$, respectively. Some viral transport media, used routinely to stabilise RNA in clinical samples during transportation, caused a non-specific colour-change in the LAMP reaction and therefore we suggest collecting samples in phosphate buffered saline (which did not affect the colour) as the assay allows immediate sample analysis on-site.

\section{Conclusion}

SARS-CoV-2 LAMP is a cheap and reliable assay that can be readily employed in RLS to improve disease monitoring and management.

\section{Background}


Two years after the outbreak of the Severe Acute Respiratory Syndrome Coronavirus 2 (SARS-CoV-2) the number of people infected with this new coronavirus is approaching 340 million and more than 5.5 million have died (as of January 2022) (1). While great international efforts have led to the development and approval of highly effective vaccines against SARS-CoV-2 (2) vaccine-breakthroughs are very common $(3,4)$, many countries are still seeing high infection rates and are experiencing new waves of infection. To minimise the spread of infection fast isolation of infected individuals as well as efficient and accurate testing is essential. The current gold-standard diagnostic assay for SARS-CoV-2 is a quantitative reverse-transcription PCR (qRT-PCR) assay, which has been developed and optimised in different reference laboratories, including Berlin Germany, CDC China and CDC USA (5-7), and subsequently received approval from the FDA ( 8).

Fast roll-out of testing was achieved in Europe, North America and Asia with a minimum of $>2,000$ tests per million individuals to date (December 2021) and Austria even reaching a testing capacity of 46,000 per $10^{6}$ people. In Africa the testing rate is currently well below 500 tests per million individuals (9), largely because contributing factors include the lack of specialised laboratories and trained staff, insufficient infrastructure for sample transportation and communication of results back to the patient, high costs of the assay and a worldwide shortage of PCR reagents. Therefore, a sensitive, specific and cheap SARS-CoV-2 assay, which does not require the sample to be sent to a specialised laboratory, is urgently needed.

Lateral Flow Tests based on the detection of SARS-CoV-2 antigens have been approved for detection of infections (10). Although these tests can be used at home they are a lot less sensitive compared to qRTPCR, with their accuracy being highest when individuals are symptomatic ( $72 \%$ accuracy), decreasing to $58 \%$ in asymptomatic people (11). As this type of test is designed for self-sampling, this adds the risk of inappropriate sample taking by untrained people thus further reducing the accuracy of the test. Therefore, in many countries these tests require additional confirmation by qRT-PCR before a positive test is registered.

The loop-mediated isothermal amplification assay (LAMP) is a rapid and very specific diagnostic assay (12) that can be used as a point-of-care (POC) test and can give results within 20 to 30 minutes after taking the sample. Reagents are readily available. The colourimetric assay is based on nucleic acidamplification and uses the fact that during incorporation of dNTPs into newly synthesised DNA H+ ions are released and lead to acidification of the reaction solution. This can be made visible with Phenolred, which turns from a pink colour in basic environments (at the start of the reaction) to a yellow colour in acidic solutions (at the end of the reaction after RNA-amplification) (13). Fluorescent LAMP assays use a fluorescent dye, which intercalates in double stranded DNA and can be detected, for example, with a light cycler.

For SARS-CoV-2 several LAMP assays have been developed which give reliable results and have received FDA emergency use and authorisation as a POC test (14). In our study we compared the sensitivity of different SARS-CoV-2 LAMP primers with qRT-PCR and tested the applicability of the assay in Malawi as 
an example of a resource limited country. We show that the LAMP assay is specific when compared to qRT-PCR. It is a rapid method (30 minutes), which requires minimal equipment and training, has been successfully tested at Kamuzu College of Health Sciences (KUHeS) in Blantyre, Malawi and its teaching institution Queen Elizabeth Central hospital (QECH). It is cheaper than qRT-PCR and can therefore easily be introduced as POC in resource-limited settings.

\section{Methods}

\section{Samples and ethical statement:}

Residual samples from St. George's Hospital in London, Great Ormond Street Hospital NHS Foundation Trust in London (collected March-May 2020) and QECH in Malawi (collected June-July 2020) were used for LAMP test validation. Ethics approval for KUHeS was obtained from the College of Medicine Research Ethics Committee (COMREC) as part of LAMP assay development studies. Anonymised residual samples originating from the UK were used in accordance with the Human Tissue Act and the RCPath guidelines for assay development and validation.

\section{Aim, design and setting of the study:}

The aim of the study was to designt and optimise a SARS-CoV-2 LAMP assay as rapid test for POC testing in LMICs. At the Institute of Child Health, London, extracted and non-extracted SARS-CoV-2+ samples (identified with qRT-PCR) were used for the optimisation of the LAMP assay, which was afterwards rolled out in Malawi to retrospectively test 92 non-extracted SARS-CoV-2 samples. Results suggest applicability of the SARS-CoV-2 LAMP assay as rapid POC test in LMICs.

\section{RNA extraction and qRT-PCR:}

RNA was extracted from 200 $\mu$ l of swab sample. At KUHeS RNA extracted using the Omega Biotek Magbind Viral DNA/RNA Kit according to the manufacturer's protocol. The 2019-nCOV CDC EUA Kit primers in combination with qScript ${ }^{\mathrm{TM}}$ XLT 1-Step RT-qPCR ToughMix Low ROX mastermix from Quantabio were used for the qRT-PCR. The qRT-PCR was performed on the Quantstudio 7 Flex PCR system.

At St. George's Hospital the Magna Pure 96 DNA and Viral NA Small Volume Kit 2.0 (Roche) was used

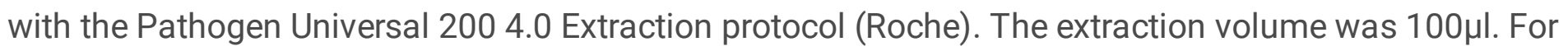
the qRT-PCR $10 \mu$ l of extract was tested for SARS-CoV-2 using the Altona Diagnostics Real Star SARS-CoV2 RT-PCR kit 1.0 on the Roche Light Cycler 480, according to the manufacturer's protocol.

\section{Colourimetric LAMP assay:}

Primers are listed in Supplementary Table S1. The protocol from the New England Biolab (NEB) colourimetric LAMP assay was followed with slight modifications to test patient samples. Briefly, all reagents were thawed on ice and pipetted at room temperature. Sample input varied between $1 \mu \mathrm{l}$ and $3 \mu \mathrm{l}$ per $20 \mu \mathrm{l}$ reactions, which were performed at $63^{\circ} \mathrm{C}$ in a T100 Thermo Cycler (BioRad) in London and a 
GeneAmp PCR System 2700 (Applied Biosystems) in Malawi. A change of colour from pink to yellow indicated a positive reaction.

Samples were directly tested without prior RNA extraction were heat-inactivated at $95^{\circ} \mathrm{C}$ for 5 minutes before analysis.

\section{One-step Dpcr}

Digital droplet PCR (dPCR) was carried out using the Biorad One-Step RT dPCR Supermix and the Biorad Automated Droplet Generator for droplet generation. After the PCR reaction the droplets were read on the QX100 Droplet Reader and results analysed with the QuantaSoft Software (Biorad). Primers sequences are listed in Supplementary Table S1 and PCR reaction mix and cycling conditions were according to the manufacturer's protocol.

\section{Results}

\section{Optimisation of the colourimetric LAMP assay}

Four different LAMP primer sets that bind to different regions of the SARS-CoV-2 genome (primer sequences see Supplementary Table S1) were tested on extracted SARS-CoV-2 RNA samples that had previously been tested with qRT-PCR at St. George's University Hospital in London. We chose published primers targeting orf1a, $N(15)$ and orf1ab (16). Additionally, we also adapted primers from Hong et al., which bind to the replicase open reading frame (orf1ab) (17) of SARS-CoV-1, to detect SARS-CoV-2. One microliter of five positive and three negative samples (determined by qRT-PCR) were tested at $65^{\circ} \mathrm{C}$ following the manufacturer's protocol and colour-change was monitored every ten minutes up to 60 minutes. The primer set adapted from SARS-CoV-1 (17) did not give any positive results in the colourimetric LAMP assay (data not shown). Figure 1A shows the results for the three published primer sets for SARS-CoV-2 $(15,16)$. The best results were obtained with the N-primers, which detected 4 of 5 positive samples, both orf 1 primer sets were less sensitive and detected only 3 of 5 and 1 of 5 positive samples (Figure 1A).

Next we tested the N-primers in a temperature gradient ranging from $59^{\circ} \mathrm{C}$ to $67^{\circ} \mathrm{C}$, again observing the reaction every 10 minutes up to 60 minutes to see how the LAMP assay performs at a wider temperature range. At the same time we also compared sample input of 1 and $3 \mu l$ at $63^{\circ} \mathrm{C}$ and $65^{\circ} \mathrm{C}$. The fastest colour change was seen at $63^{\circ} \mathrm{C}$, appearing after 20 minutes, closely followed by $65^{\circ} \mathrm{C}$, which is the optimal temperature given by the manufacturer (Figure 1B). Robust colour change was seen at temperatures between $61^{\circ} \mathrm{C}$ and $67^{\circ} \mathrm{C}$, indicating a relatively wide temperature range at which the LAMP assay can be performed. The lowest temperature of $59^{\circ} \mathrm{C}$ did not show a colour change. The amount of sample input also seems to be important, because the colour reaction with $3 \mu$ sample was not as clear as with $1 \mu$ sample, which may indicate inhibitory effects due to large amounts of nucleic acids being present in the reaction. 
The ideal condition to perform the LAMP assay seems to be the $N$ primer set from Zhang et al. (15) at a temperature of $63^{\circ} \mathrm{C}$ for 30 to 40 minutes (N-LAMP). Since the orf1ab primer set from Yu et al. (16) also performed well (Orf-LAMP) it was in parallel also used to test clinical samples for for the presence of SARS-CoV-2.

To determine the approximate limit of detection of the SARS-CoV-2 N-LAMP assay we determined the copy number of two of our samples in a One-step dPCR reaction using the N2-primers that have been published by the CDC (18). Both samples were then serially diluted from $10^{\wedge} 4$ to $10^{\wedge} 0$ copy numbers per reaction in five technical replicates. Figure $1 \mathrm{C}$ shows that the limit of detection for the N-LAMP assay lies at approximately 100 copies per reaction.

\section{Specificity and Sensitivity of the colourimetric LAMP assay compared to diagnostic qRT-PCR}

We tested 92 RNA-extracted swab samples from St. George's University Hospitals in the LAMP assay that had previously been tested by qRT-PCR using envelope and spike primers as well as qRT-PCR using the CDC N-primers (18). Figure 2 and Supplementary Figure S1 shows that with either the N-or the Orf-LAMP no false-positive samples were detected. Therefore, the specificity of both LAMP assays compared to qRT-PCR was $100 \%$.

The sensitivity of the LAMP assay was found to be slightly lower compared to qRT-PCR (73\% for N-LAMP and $62 \%$ for Orf-LAMP), as both LAMP primer pairs missed some qRT-PCR positive samples (Figure 2). This was probably due to low amounts of RNA in those samples as shown by a high Ct value of $>30$ in the qRT-PCR assays.

\section{Use of inactivated non-extracted samples in the colourimetric LAMP assay}

To simplify the assay further, shorten the turn-around time and reduce costs we tested the $\mathrm{N}$ - and OrfLAMP assay on non-extracted samples that had previously been tested for SARS-CoV-2 at Great Ormond Street Hospital in London. Ten positive and two negative clinical samples collected in phosphate buffered saline (PBS) were tested using both, the N-LAMP and Orf-LAMP assays (Figure 3A). The N-LAMP was again more sensitive than the Orf-LAMP assay, detecting 8 out of 10 and 3 out of 10 positive samples, respectively. We further tested 35 non-extracted longitudinal swab samples from three patients infected with the SARS-CoV-2 Delta variant. Viral nucleic acids could be detected with the N-LAMP assay in positive samples (data not shown).

\section{Compatibility of various viral transport media with the LAMP assay}

Various viral transport media (VTM) are currently being used worldwide to preserve RNA within clinical specimens during transportation and prior to sample analysis. As the colourimetric LAMP assay measures a pH change caused by the release of $\mathrm{H}+$ ions during the synthesis of new DNA we assessed 
whether different VTMs on their own would already influence the $\mathrm{pH}$ in the reaction mix without incubation at $63^{\circ} \mathrm{C}$. Addition of Universal Transport Medium (UTM, MANTACC), Medical Wire viral medium (MWE) and BDS Sample Preservation Solution were incompatible with the colourimetric LAMP assay, resulting in a colour change from red to yellow immediately after adding to the reaction mix (data not shown). Dewei VTM (Dewei) spiked with SARS-CoV-2 RNA showed inhibition of DNA synthesis (no colour change) in the LAMP reaction, however, heat-inactivation of the Dewei samples for 5 minutes at $95^{\circ} \mathrm{C}$ resulted in a positive reaction seen as colour change from pink to yellow (Figure 3B). Heatinactivated samples collected in PBS and Beaver VTM (Beaver Biomedical Engineering Co) were also compatible with the LAMP reaction mix (data not shown).

\section{Testing of the LAMP assay at KUHeS in Malawi}

To assess the handling, feasibility and rapidity of the N-LAMP assay in a resource-limited setting (RLS) we tested 92 non-extracted samples with known qRT-PCR result directly on site at KUHeS in Malawi. The results are shown in Figure 4. The LAMP assay performed with a specificity of $98.4 \%$ and a sensitivity of $86.7 \%$, compared to qRT-PCR. Again, samples with very high Ct values (above 33 ) tended to be negative in the N-LAMP assay. Assay inhibition was seen with high amounts of RNA (low Ct-value in qRT-PCR) resulting in a false negative result. Diluting the sample 1:10 resolved this issue and these samples subsequently tested positive by N-LAMP. This confirms that the amount of nucleic acid input into the LAMP assay is important (compare to Figure 1B).

Interestingly, we found one sample that was negative in qRT-PCR but positive in both, the N-LAMP and Orf-LAMP, in two replicates (Figure 4). This was very surprising and this sample needs further characterisation (e.g. sequencing) to determine whether this is a contamination or a mutant that is not detected by qRT-PCR.

\section{Discussion}

The current study confirms that colourimetric SARS-CoV-2 LAMP is a fast, sensitive and reliable assay, which does not require any expensive or bulky equipment. The assay can readily be adapted for use in a RLS such as Malawi and therefore could significantly impact on the local SARS-CoV-2 testing capacity.

Although infection rates in Sub-Saharan Africa seem to be lower than elsewhere, testing levels have also generally been lagging behind those in developed economies. This makes clear predictions of the true number of Coronavirus disease 2019 (COVID-19) cases and deaths difficult. A post-mortem study conducted in Zambia showed that due to the lack of SARS-CoV-2 testing, particularly in the wider community, a large number of deaths associated with COVID-19 were missed (19). Similarly, Mulenga et al. reported that for every 92 SARS-CoV-2 infections in the community only 1 laboratory-confirmed case was reported (20). Fast, reliable, easy-to-use and affordable SARS-CoV-2 tests are key for monitoring the spread of disease in communities to provide appropriate care, prevent further transmission and allowing the informed management of interventions, such as local lockdowns and implementation of social distancing. SARS-CoV-2 LAMP assays have been described as a low-cost molecular alternative to qRT- 
PCR $(21,22)$ and are used in developed economies as diagnostic tests. However, little is known about their utilisation in RLSs. Baba et al. reported the feasibility of a SARS-CoV-2 LAMP assay in Cameroon, Ethiopia and Nigeria (23). Their test included a RNA-extraction step, which significantly increases costs, requires a specialised laboratory and lengthens the turn-around-time.

The advantage of the assay described in our study is firstly the direct use of heat-inactivated samples, eliminating lengthy and costly RNA extraction and reducing the risk of infection when samples are handled. Indeed, the assay was more sensitive when samples were not extracted, which may be due to loss of RNA during the extraction process. Secondly, a water-bath or heat-block is sufficient and as the reaction can tolerate a temperature range from $61-67^{\circ} \mathrm{C}$ it is less sensitive to temperature fluctuations that may occur in RLSs due to unstable electricity supply. Thirdly, in contrast to standard qRT-PCR, results are available within 30 minutes after the sample was taken. In addition, reagents are readily available and are much cheaper at a cost of approximately $£ 3$ compared to $£ 30$ for qRT-PCR.

Of the two primer sets that we tested, the N-LAMP was more sensitive compared to the Orf-LAMP. This is likely due to N RNA being the highest expressed SARS-CoV-2 RNA during virus replication (24).

We found that the amount of input-RNA is critical. High RNA concentration seems to inhibit the reaction, causing false-negative results. To our knowledge, this is the first description of inhibition of a LAMP reaction by very high nucleic acid concentrations. We showed that performing the LAMP assay with undiluted and 1:10 diluted sample resolved this issue.

The SARS-CoV-2 Delta variant could readily be detected by the N-LAMP assay, indicating a higher stability of the assay towards new variants, but further testing and validation of new occurring variants is required.

Limitations of the SARS-CoV-2 LAMP assay include the influence of different viral transport media on the $\mathrm{pH}$ of the reaction. Buffers or viral transport media need to be chosen carefully before performing LAMP. We found that collecting samples in PBS is the simplest method, providing the samples are processed fairly quickly. As the LAMP assay is intended to be used as a point-of-care test neither storage nor transportation of the samples is necessary.

Compared to qRT-PCR the LAMP assays were about 10-100fold less sensitive ((25) and this work). SARSCoV-2 RNA can be detected in infected individuals even before symptom onset, which infers that individuals could already spread the virus when still asymptomatic, as well as during the symptomatic phase (26), but the detection of infectious virus seems to wane within the first two to three weeks after infection $(26,27)$. During this phase SARS-CoV-2 RNA in patients is very high and LAMP should be more than adequate to detect the virus and especially quarantine asymptomatic people.

\section{Conclusion}


The optimised assay showed similar sensitivity and specificity in Malawi and in London. Future prospective studies in rural health care centres should establish whether this test can be used for wider community surveillance, to inform on adequate disease management.

\section{List Of Abbreviations}

COVID-19 Coronavirus Disease 19

dPCR droplet polymerase chain reaction

LAMP loop-mediated isothermal amplification

MWE $\quad$ Medical Wire viral medium

PBS phosphate buffered saline

POC point-of-care

qRT-PCR quantitative reverse transcription polymerase chain reaction

RLS resource limited settings

SARS-CoV-2 Severe Acute Respiratory Syndrome Coronavirus 2

UTM universal transport medium

VTM viral transport medium

\section{Declarations}

\section{Ethical approval:}

Ethics approval for KUHeS was obtained from the College of Medicine Research Ethics Committee (COMREC) as part of LAMP assay development studies. Anonymised residual samples originating from the UK were used in accordance with the Human Tissue Act and the RCPath guidelines for assay development and validation.

\section{Consent for publication:}

Not applicable.

\section{Availability of data and materials:}

Original datasets can be obtained from the corresponding author on reasonable request.

\section{Competing interests:}


The authors declare no competing interests related to this work.

\section{Funding:}

This work was partially funded by the Reuben's Centre for Virology and Metagenomics.

\section{Authors' contributions:}

KG, DGA wrote the manuscript; KG, DGA, NK developed the study design; KG, HM, MK, LS, MP, DGA, EC-G performed the experiments, KG, NK, DGA, HM, MN-N, TN interpreted the data; all authors commented on the manuscript.

\section{Acknowledgements:}

We are grateful to Great Ormond Street Hospital NHS Foundation Trust London, UK and St. George's University Hospitals London, UK and KUHeS, Malawi for providing us with validation samples.

\section{References}

1. John's Hopkins University Coronavirus Resource Centre. 2020 [Available from: https://coronavirus.jhu.edu.

2. Samaranayake LP, Seneviratne CJ, Fakhruddin KS. Coronavirus Disease 2019 (COVID-19) Vaccines: A Concise Review. Oral Dis. 2021.

3. Butt AA, Nafady-Hego H, Chemaitelly H, Abou-Samra AB, Khal AA, Coyle PV, et al. Outcomes Among Patients with Breakthrough SARS-CoV-2 Infection After Vaccination. Int J Infect Dis. 2021;110:3538.

4. Hacisuleyman E, Hale C, Saito Y, Blachere NE, Bergh M, Conlon EG, et al. Vaccine Breakthrough Infections with SARS-CoV-2 Variants. N Engl J Med. 2021;384(23):2212-8.

5. Institute of Viral Diseases. China CDC. National Institute for Viral Disease Control and Prevention $2020[$.

6. Coronavirus Disease 2019 (COVID-19). Centers for Disease Control and Prevention. https://wwwcdcgov/coronavirus/2019-ncov/lab/rt-pcr-panel-primer-probeshtml. 2020.

7. Corman VM, Landt O, Kaiser M, Molenkamp R, Meijer A, Chu DK, et al. Detection of 2019 novel coronavirus (2019-nCoV) by real-time RT-PCR. Euro Surveill. 2020;25(3).

8. FDA approved SARS-CoV-2 diagnostic tests 2020 [Available from: https://www.fda.gov/emergencypreparedness-and-response/mcm-legal-regulatory-and-policy-framework/emergency-useauthorization\#covidinvitrodev.

9. https://ourworldindata.org/coronavirus-testing. 2021.

10. Katyal P, Mahmoudinobar F, Montclare JK. Recent trends in peptide and protein-based hydrogels. Curr Opin Struct Biol. 2020;63:97-105. 
11. Dinnes J, Deeks JJ, Berhane S, Taylor M, Adriano A, Davenport C, et al. Rapid, point-of-care antigen and molecular-based tests for diagnosis of SARS-CoV-2 infection. Cochrane Database Syst Rev. 2021;3:CD013705.

12. Notomi T, Okayama H, Masubuchi H, Yonekawa T, Watanabe K, Amino N, et al. Loop-mediated isothermal amplification of DNA. Nucleic Acids Res. 2000;28(12):E63.

13. Tanner NA, Zhang Y, Evans TC. Jr. Visual detection of isothermal nucleic acid amplification using pHsensitive dyes. Biotechniques. 2015;58(2):59-68.

14. FDA. Emergency use authorization (EUA) summary for the color SARS-CoV-2 RT-LAMP diagnostic assay. https://wwwfdagov/media/138249/download. 2021.

15. N.A. ZYONXJSLNROWHT. Rapid Molecular Detection of SARS-CoV-2 (COVID-19) Virus RNA Using Colorimetric LAMP. MedRxiv. 2020.

16. Yu LW, Hao S, Li X, Liu X, Ye X, Han S, Dong H, Li X, Li X, Liu J, Liu N, Zhang J, Pelechano W, Chen VWei-Hua, Yin W-H, X. Rapid colorimetric detection of COVID-19 coronavirus using a reverse transcriptional loop-mediated isothermal amplification (RT-LAMP) diagnostic plat- form: iLACO. medRxiv. 2020.

17. Hong TC, Mai QL, Cuong DV, Parida M, Minekawa H, Notomi T, et al. Development and evaluation of a novel loop-mediated isothermal amplification method for rapid detection of severe acute respiratory syndrome coronavirus. J Clin Microbiol. 2004;42(5):1956-61.

18. Coronavirus Disease 2019 (COVID-19). Centers for Disease Control and Prevention 2020 [.

19. Mwananyanda L, Gill CJ, MacLeod W, Kwenda G, Pieciak R, Mupila Z, et al. Covid-19 deaths in Africa: prospective systematic postmortem surveillance study. BMJ. 2021;372:n334.

20. Mulenga LB, Hines JZ, Fwoloshi S, Chirwa L, Siwingwa M, Yingst S, et al. Prevalence of SARS-CoV-2 in six districts in Zambia in July, 2020: a cross-sectional cluster sample survey. Lancet Glob Health. 2021;9(6):e773-e81.

21. Chaouch M. Loop-mediated isothermal amplification (LAMP): An effective molecular point-of-care technique for the rapid diagnosis of coronavirus SARS-CoV-2. Rev Med Virol. 2021;31(6):e2215.

22. Shabani E, Dowlatshahi S, Abdekhodaie MJ. Laboratory detection methods for the human coronaviruses. Eur J Clin Microbiol Infect Dis. 2021;40(2):225-46.

23. Baba MM, Bitew M, Fokam J, Lelo EA, Ahidjo A, Asmamaw K, et al. Diagnostic performance of a colorimetric RT -LAMP for the identification of SARS-CoV-2: A multicenter prospective clinical evaluation in sub-Saharan Africa. EClinicalMedicine. 2021;40:101101.

24. Kim D, Lee JY, Yang JS, Kim JW, Kim VN, Chang H. The Architecture of SARS-CoV-2 Transcriptome. Cell. 2020;181(4):914-21 e10.

25. Mautner L, Baillie CK, Herold HM, Volkwein W, Guertler P, Eberle U, et al. Rapid point-of-care detection of SARS-CoV-2 using reverse transcription loop-mediated isothermal amplification (RT-LAMP). Virol J. 2020;17(1):160. 
26. Woudenberg T, Eberle U, Marosevic D, Liebl B, Ackermann N, Katz K, et al. Detection and viral RNA shedding of SARS-CoV-2 in respiratory specimens relative to symptom onset among COVID-19 patients in Bavaria, Germany. Epidemiol Infect. 2021;149:e150.

27. Wolfel R, Corman VM, Guggemos W, Seilmaier M, Zange S, Muller MA, et al. Virological assessment of hospitalized patients with COVID-2019. Nature. 2020;581(7809):465-9.

\section{Figures}


A) qRT-PCR: $\mathrm{neg}++\cdot++++$
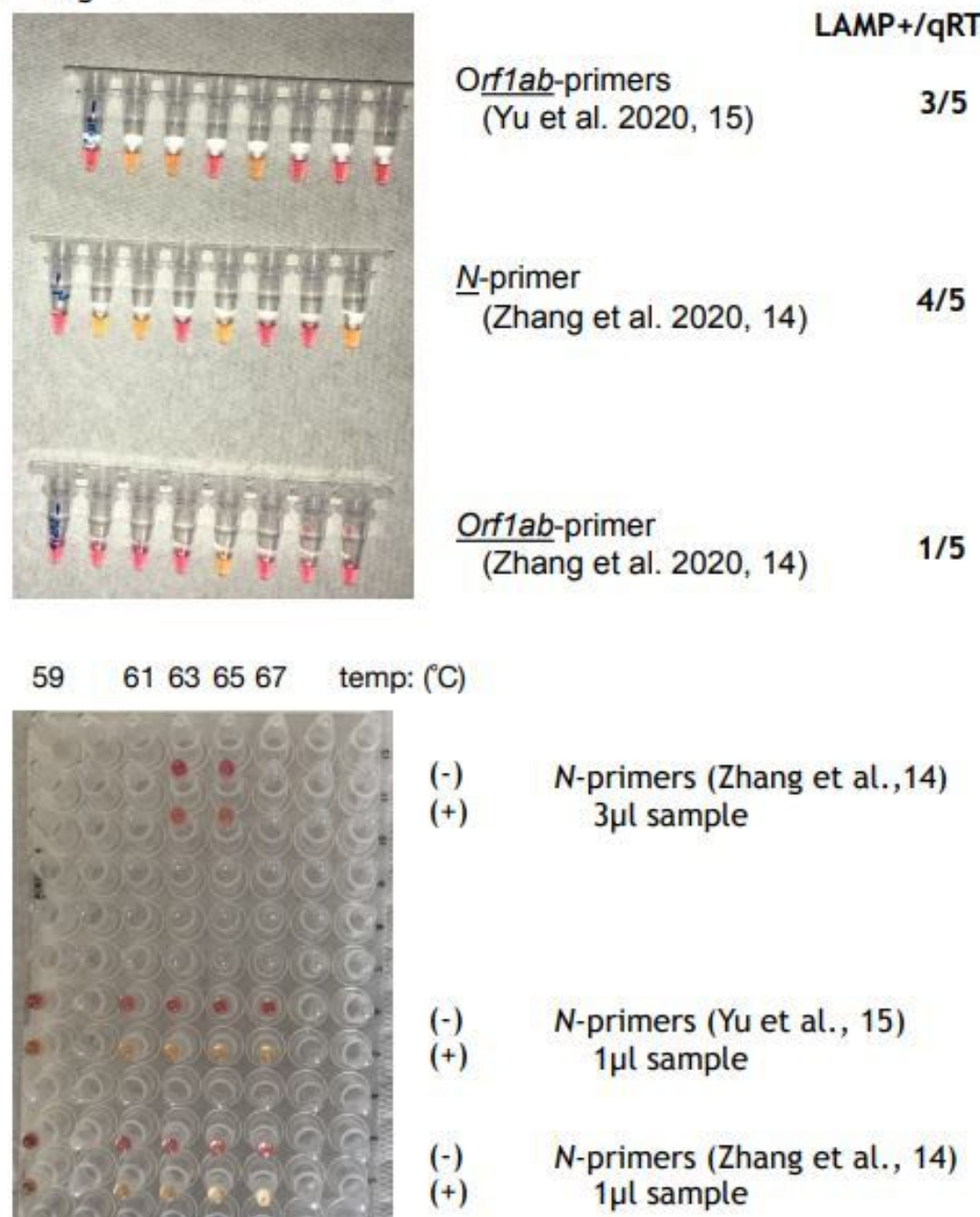

(-) $\quad \mathrm{N}$-primers (Zhang et al.,14)

(+) $\quad 3 \mu \mathrm{l}$ sample

(-) N-primers (Yu et al., 15)

(+) $1 \mu \mathrm{l}$ sample

(-) N-primers (Zhang et al., 14)

(+) $1 \mu \mathrm{l}$ sample

B)

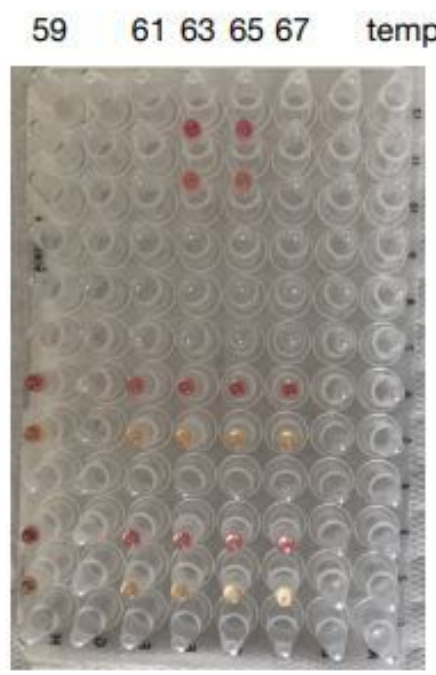

C)

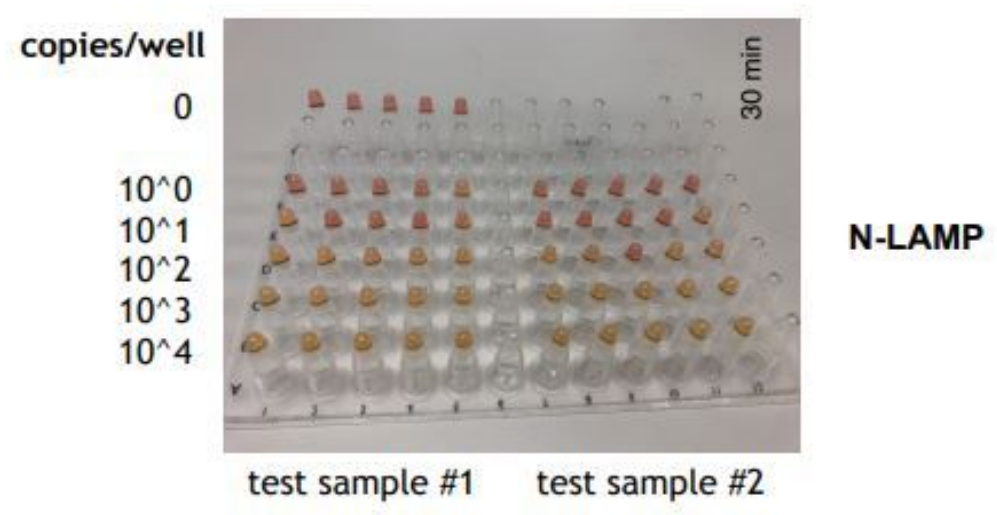

Figure 1

A) Comparison of different primers on 5 positive and 2 negative qRT-PCR samples (taken from different publications as indicated), neg: $\mathrm{H} 2 \mathrm{O}$ control; B) Temperature gradient to test LAMP stability, results were recorded after 30min; C) Determination of LAMP sensitivity. 
A)

N-LAMP assay

\begin{tabular}{|c|c|c|c|}
\hline \multirow{4}{*}{ qRT-PCR } & pos & 33 & 12 \\
\cline { 2 - 4 } & neg & 0 & 48 \\
\cline { 2 - 4 }
\end{tabular}

time to result

Sensitivity $73 \%$

Specificity: 100\%

$30 \mathrm{~min}$

B)

\section{Orf-LAMP assay}

\begin{tabular}{|c|c|c|}
\hline & pos & neg \\
\hline pos & 28 & 17 \\
\hline neg & 0 & 48 \\
\hline
\end{tabular}

Sensitivity $62 \%$

Specificity: 100\%

\section{Figure 2}

Sensitivity and specificity of LAMP assay compared to qRT-PCR on samples from St. George's Hospital, London; A) N primers and B) Orf1ab primers from Yu et al. (16) 
A)

\begin{tabular}{|c|c|c|c|}
\hline Sample no & GOSH qRT-PCR & N-LAMP & Orf-LAMP \\
\hline 1 & + & + & + \\
\hline 2 & + & - & - \\
\hline 3 & + & + & - \\
\hline 4 & + & - & - \\
\hline 5 & + & + & - \\
\hline 6 & + & + & + \\
\hline 7 & + & + & - \\
\hline 8 & + & + & - \\
\hline 9 & + & + & + \\
\hline 10 & + & + & - \\
\hline 11 & - & - & - \\
\hline 12 & - & - & - \\
\hline
\end{tabular}

specificity compared to qRT-PCR:

$\begin{array}{ccc}8 / 10 & 3 / 10 & \text { positive } \\ 2 / 2 & 2 / 2 & \text { negative }\end{array}$

B)

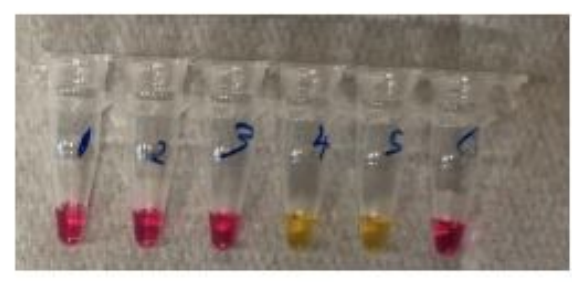

\section{N-LAMP}

\section{RNA}

heat-inactivated

VTM buffer

\section{Figure 3}

N-LAMP of non-extracted swab samples from GOSH, London (30min incubation); A) results for 12 nonextracted patient samples; B) N-LAMP assay of RNA-spiked samples to test influence of VTM buffer (heat- or non heat-inactivated). 


\section{N-LAMP assay}

\begin{tabular}{|c|c|c|c|c|}
\hline \multirow{3}{*}{ qRT-PCR } & & pos & neg & \multirow[b]{2}{*}{ Sensitivity $86.7 \%$} \\
\hline & pos & 26 & 4 & \\
\hline & neg & 1 & 61 & Specificity $98.4 \%$ \\
\hline
\end{tabular}

Figure 4

Results for N-LAMP testing of non-extracted swab samples at KUHeS, Malawi

\section{Supplementary Files}

This is a list of supplementary files associated with this preprint. Click to download.

- SupplementaryTableS1.docx

- SupFig1.jpg 